\title{
OGLE II Observations of Star Clusters in the Magellanic Clouds
}

\author{
G. Pietrzyński \\ Universidad de Concepción, Departamento de Fisica,Casilla 160-C, \\ Concepción, Chile \\ Warsaw University Observatory, Al. Ujazdowskie 4,00-478, Warsaw
}

\begin{abstract}
In the course of the OGLE II experiment we have collected up to now about one billion BVI observations of ten million stars in the Magellanic Clouds. Based on that data about 1000 star clusters were detected with an automatic algorithm. In a series of papers we published catalogs of clusters, multiple cluster candidates and variable stars located in the fields of detected clusters. We also presented analysis of the properties of detected clusters. This year the new phase of the OGLE project - OGLE III - has started. With the new mosaic detector we will increase the data flow by about an order of magnitude.
\end{abstract}

\section{Introduction}

The Optical Gravitational Lensing Experiment (OGLE) is a long term observing program with the main goal of probing dark matter in the Galaxy with microlensing phenomena (Paczyński 1986). Because of the very low probability of microlensing detection, a lot of stars need to be observed over a long period of time, to provide a statistically significant sample. In the course of the second phase of this project (OGLE II) about 40 million stars were monitored every clear night during 4 years. The resulting huge database of about one billion high quality B V I measurements provide a unique database, very well suited for many other subprojects unrelated to microlensing. One such subproject aims at searching and analysing the properties of star clusters in the Magellanic Clouds.

\section{Observations}

All presented observations were obtained during the OGLE II project with the $1.3 \mathrm{~m}$ Warsaw telescope at the Las Campanas Observatory, Chile (Udalski, Szymański and Kubiak 1997). Twenty six and 11 driftscan fields, with a size of about 14.5 arcmin $\times 57$ arcmin each, were monitored in the LMC and the SMC, respectively. The resulting database (Udalski et al. 1997, 2000) comprises about 10 million stars. Typically about $400 \mathrm{I}, 15 \mathrm{~V}$ and $15 \mathrm{~B}$ observations were made for each star. The photometry is complete down to 21.5 mag. The accuracy of the zero point is better than 0.02 mag. 


\section{Catalogs of Clusters}

An automatic, algorithmic method was applied for searching for star clusters. Briefly, for each driftscan a density map was prepared and then "unsharp masked" in order to remove smooth variations. Then the potential clusters were selected as concentrations of at least four pixels exceeding a threshold of $2 \sigma$. This procedure resulted in the detection of 273 and 745 star clusters in the SMC (Pietrzyński et al. 1998) and the LMC (Pietrzyński et al. 1999), respectively.

\section{Size and Age Distributions}

The size distributions of clusters from the LMC and the SMC has a very similar shape. We find that the SMC contains more large clusters which may be explained by the weaker gravitational field of the SMC.

Ages were derived for about 700 clusters (Pietrzyński and Udalski 1999a, 2000a). The age distribution of the LMC clusters reveals a bursty nature of cluster formation in this galaxy, in contrast to the relatively uniform distribution of cluster ages in the SMC. We detected three extended peaks in the age distribution of LMC clusters, located at about $0.8 \mathrm{Gyr}, 125 \mathrm{Myr}$ and $7 \mathrm{Myr}$. All detected peaks have a complex structure. While the structure of the youngest and the oldest peaks may be spurious due to the limited accuracy of age determination, the middle peak clearly reveals two evident subpeaks at $100 \mathrm{Myr}$ and 160 Myr.

Similar peaks are seen in the age distribution of clusters in the SMC, which indicates that an increased cluster formation rate during these periods might have been caused by the last encounter between the Magellanic Clouds. The sample of clusters from the LMC is overabundant in very young $(<10 \mathrm{Myr})$ and old ( $>400 \mathrm{Myr}$ ) clusters, and underabundant in clusters having ages in the range from 80 to $15 \mathrm{Myr}$ with respect to the studied sample of clusters from the SMC.

\section{Multiple Clusters}

One hundred and twenty seven multiple cluster candidates were selected from the OGLE catalogs of star clusters in the LMC (Pietrzyński and Udalski 1999b) and in the SMC (Pietrzyński and Udalski 2000b), respectively. A statistical analysis shows that a significant fraction of these objects may constitute physical systems. A coeval age of most of the components of multiple objects suggests their common origin. The comparison of the population of multiple cluster candidates from the SMC and LMC shows: a) the shapes of the distributions of size and age of multiple and single clusters from the Magellanic Clouds are very similar, b) the difference of size of the components of a given system is small, c) in the distributions of the separation between the components of multiple clusters both in the LMC and SMC there are two peaks at about 9 and $15 \mathrm{pc}$. 


\section{Variable Stars}

The large number of observations spanned over a long period of time provides also a unique opportunity to explore populations of variable stars from star clusters in the Magellanic Clouds. A list of 336 Cepheids and 127 eclipsing systems located in the fields of star clusters was already prepared (Pietrzyński and Udalski $1999 \mathrm{c}, \mathrm{d}$ ). Further papers on other types of variable stars in star clusters will follow.

\section{OGLE III}

The OGLE II project suspended regular observations on November 26, 2000. Observations will be resumed in 2001 as the OGLE-III phase. The major upgrade will include a new "second generation" CCD mosaic camera consisting of eight thin SITe $2048 \times 4096$ CCD chips (8192x8192 pixels of 0.26 "/pixel) giving the total field of view equal to $35^{\prime} \times 35$ '. The area regularly observed during OGLE-III will be about 8 times larger than that observed during the OGLE-II phase.

Acknowledgments. I would like to thank the Scientific Organizing Committee for partial financial support. The OGLE project is supported by the NSF grant AST-9820314.

\section{References}

Paczyński, B. 1986, ApJ, 304, 1

Pietrzyński, G. \& Udalski, A. 1999a, Acta Astron., 49, 165

Pietrzyński, G. \& Udalski, A. 1999b, Acta Astron., 49, 157

Pietrzyński, G. \& Udalski, A. 1999c, Acta Astron., 49, 149

Pietrzyński, G. \& Udalski, A. 1999d, Acta Astron., 49, 543

Pietrzyński, G. \& Udalski, A. 2000a, Acta Astron., 50, 337

Pietrzyński, G. \& Udalski, A. 2000b, Acta Astron., 50, 355

Pietrzyński, G., Udalski, A., Kubiak, M., Szymański, M., Woźniak, P., \& Żebruń, K. 1998 Acta Astron., 48, 175

Pietrzyński, G., Udalski, A., Kubiak, M., Szymański, M., Woźniak, P., \& Żebruń, K. 1999 Acta Astron., 49, 521

Udalski, A., Kubiak, M., and Szymański,M. 1997 Acta Astron., 47, 319

Udalski, A., Kubiak, M., and Szymański,M. Pietrzyński, G., Woźniak, P., \& Żebruń, K. 1998 Acta Astron., 48, 147

Udalski, A., Kubiak, M., and Szymański,M. Pietrzyński, G., Woźniak, P., \& Żebruń, K. 2000 Acta Astron., 50, 289 